\title{
Adapting The Extended Neyman's Smooth Test to Be Used in Accelerated Failure Time Models
}

\author{
Abdalla Abdel-Ghaly*, Hanan Aly and Elham Abdel-Rahman \\ Cairo University
}

\begin{abstract}
Accelerated life testing (ALT) has gained greater importance because of dealing with high reliability units. As a result, there is a big need to use a goodness of fit (GOF) technique for testing the underlying lifetime distribution. But there is a difficulty due to the existence of several stress levels with different samples of units at each level. Then, the choice of a certain GOF technique is based on its capability to combine the failure times from all stress levels to reach a conclusion about the adequacy of a certain lifetime distribution at each stress level.

In this paper, the extended Neyman's smooth test (ENST) is chosen. It is then modified in order to be used in validating the distributional assumption of accelerated failure time (AFT) model. This modified method is called; the adapted extended Neyman's smooth test (AENST). It is applied to test for both Weibull and exponential distributions in case of constant stress under complete sampling. To check the performance of the AENST, a comparison is made with the conditional probability integral transformation test (CPITT) via a simulation study. Moreover, a real data set is provided to illustrate the application of the introduced AENST. The results revealed that the AENST is a powerful test comparing with the CPITT. Thus, the AENST is recommended for testing the AFT models.
\end{abstract}

Key words: Accelerated life testing, Accelerated failure time model, Constant stress, Goodness of fit tests, Extended Neyman's smooth test, Partial scores, Conditional probability integral transformation test.

\section{Introduction}

Global competition in companion with using higher manufacturing technologies resulted in producing high reliability products. Testing whether these products are sufficiently reliable to be utilized may require long time. This led to the use of accelerated life testing (ALT) in today's industry in order to obtain failures more quickly. Under ALT, items are subjected to stresses at several levels higher than those experienced under normal conditions so as to obtain failures under normal levels. This can be achieved via using accelerated life models (ALM) that relate

* Corresponding author. 
the lifetime distribution to the stress. The difference between the ALM proposed in the literature is in the influence of the applied stress on the reliability (for more details, see Bagdonavičius and Nikulin (2002)).

The commonly used model in reliability analysis is the accelerated failure time (AFT) model. Under this model, increasing the stress level has the effect of shrinking time through a scale factor (For more details, see Meeker and Escobar (1993)); that is the stress affects the scale parameter of the lifetime distribution through a certain relationship. The commonly used relationships are the Arrhenius and the inverse power law relationships.

There is a great amount of literature on estimating the parameters of the lifetime distribution in the case of constant stress. This literature treats a variety of models (distributions and relationships), approaches of estimation, and different types of censoring. For example, see Abdel Ghaly et al. (1998), Drop and Mazzuchi (2004), Escobar and Meeker (2006), Liu and Tang (2009), and Abdel Ghaly et al. (2015).

Most of work in ALT assumes that the lifetime distribution is known in advance and the problem is to estimate the parameters of this distribution under the normal operating conditions. Actually, if the underlying distribution of the lifetime data does not coincide with the hypothesized one, the results drawn from ALT are nonsense and misleading. Thus, it is essential to check for the underlying lifetime distribution of the lifetime data before extracting any results from the test. This problem is known as goodness of fit (GOF) testing problem and there are some techniques proposed in the literature concerning solving this problem.

GOF tests proposed in the field of ALT can be divided into two types; the first one for ALM and the other to validate the assumptions of the AFT model. Since ALM are proposed to model the effect of the stress on the lifetime distribution, they can be thought of as some hypotheses about the influence of the applied stress on the reliability. Accordingly, some GOF techniques are proposed to assess these hypotheses. (For more details, see Bagdonavi cius and Nikulin (2002), Bagadonavičius et al. (2004), Bagadonavičius et al. (2011), and Balakrishnan et al. (2013)).

When applying GOF tests for the assumptions of an AFT model, it is assumed that the AFT model holds and GOF techniques are proposed to validate its underlying assumptions; which are

1. The life-stress relationship.

2. The family of distributions to which the failure time at each stress level belongs.

In the case of testing for the life-stress relationship, Nelson (1990) used the F statistic to test the linear life-stress relationship for $\log$-failure time variable $(\log T)$, where $T$ was assumed to have exponential, Weibull and lognormal distributions. Lawless (2003) dealt with the same case but using likelihood ratio test (LRT). Eguchi (1992) investigated the validity of the inverse power law relationship assuming a bivariate exponential distribution using a test statistic based on a projection method. Teng and Yeon (2002) proposed D-statistic based on the transformed least square (LS) estimation approach to assess the validity of the log-linear life-stress relationship 
against the log-quadratic one in case of step stress ALT experiment under exponential type II censored data.

Regarding testing for the underlying distribution at each stress level, there is no much work in the literature. This little work was based on some traditional GOF tests. For example, Sethuurman and Singpurwalla (1982) used Kolmogrov-Smirnov statistic to test that the unknown distributions at different stress levels belong to a common parametric location-scale family. Nelson (1990) used the LRT for the same case and the same distributions. Xiong and Ji (2004) used the LRT to assess the validity of the cumulative exposure model used for analyzing step stress ALT. Wang (2009) proposed a procedure based on sample spacings to test the exponentiality of the lifetime distribution for each stress level based on type II censored $k$-stages step- stress ALT in the existence of the log linear life-stress relationship. Galanova et al. (2012) and Bagadonavicius et al. (2013) discussed some theoretical problems inherent in using nonparametric GOF tests (Kolmogorov, Cramer-von Mises-Smirnov, and Anderson-Darling statistics) to validate parametric AFT models based on analyzing a sample of residuals.

Thus, there is much need to introduce other GOF tests to be used in examining the fit of certain family of distributions in AFT models. The contribution of this paper is the adaptation of the extended Neyman's smooth test (ENST) in order to validate it to be used as a GOF technique in AFT models. This test is then called; the adapted extended Neyman's smooth test (AENST). Our interest here, is on the problem of GOF pertaining to the distribution to which the lifetime at each stress level belongs. Hence, assuming that the AFT model is appropriate, we are interested in testing if the underlying distribution at each stress level belongs to a certain family. We focus on the log-location-scale family of distributions, specifically the exponential and Weibull distributions. The AENST is applied in case of constant stress and complete sampling.

Abdel-Ghaly et al. (2016) applied the conditional probability integral transfor- mation test (CPITT) in the case of AFT models. In order to check the superiority of the proposed AENST as a goodness of fit technique to be used in the case of AFT models, a numerical comparison is made between the AENST and CPITT under the same assumptions.

This paper is organized as follows. In Section 2, we present the idea on which Neyman's smooth test (NST) was built. Moreover, the extension of this test in case of composite hypothesis, named; the extended Neyman's smooth test (ENST) is also presented. In Section 3, we clarify how to adapt the ENST in order to be applicable in AFT models. This test is then called; the adapted extended Neyman's smooth test (AENST). Section 4 is devoted for summarizing the work of Abdel-Ghaly (2016) in applying the CPITT in the case of AFT models. In Section 5, a simulation study is conducted to investigate the performance of the AENST comparing with that of the CPITT. A real data set is considered in Section 6. Finally, the conclusions reached from this paper are summarized in Section 7.

\section{Neyman's Smooth and Extended Neyman's Smooth Tests}

In this Section, Neyman's smooth test (NST) and the extended Neyman's smooth test (ENST) are explained. 


\subsection{Neyman's Smooth Test (NST)}

Neyman (1937) was the pioneer to introduce the GOF smooth tests. His target was to propose a test that does not depend on the form of the null density $f_{0}(t ; \beta)$. To achieve his target, the test was based on the probability integral transformation (PIT) which implies that; if $T$ is a real-valued random variable with continuous $\operatorname{CDF} F(t ; \beta)$, then $U=F(T ; \beta)$ has a uniform distribution on the interval $(0,1)$. Thus, under NST, testing for

$$
H_{0}: f(t ; \beta)=f_{0}(t ; \beta)
$$

is equivalent to testing whether the random variable $U$ has a uniform distribution on the interval $(0,1)$. Neyman (1937) considered an alternative density to the uniform one of the form

$$
g(u ; \theta)=\exp \left\{\sum_{s=1}^{m} \theta_{s} \pi_{s}(u)-D(\theta)\right\},
$$

where $u=F(t ; \beta), D(\theta)$ is a normalizing constant, $\underline{\theta}=(\theta 1, \theta 2, \ldots, \theta m), m$ is a fixed known positive integer recommended not to exceed four, and $\pi_{s}(u)$ is the $s^{\text {th }}$ degree of Legendre polynomials.

Neyman (1937) called (2.2) the order $m$ smooth alternative density. Testing for (2.1) is equivalent to test for $H_{0}: \underline{\theta}=\underline{0}$ in the smooth alternative density and this is accomplished using a quadratic statistic in the form

$$
\Psi_{m}^{2}=\sum_{s=1}^{m} \frac{1}{n}\left\{\sum_{i=1}^{n} \pi_{s}\left(u_{i}\right)\right\}^{2} .
$$

The limiting null distribution of $\Psi_{m}^{2}$ is $\chi^{2}$ distribution with $m$ degrees of freedom. Based on the $\mathrm{p}$-value, one can judge the fit of $f_{0}(t ; \beta)$ for the data in hand. NST was proposed for fully specified distributions in the case of independent identically distributed (i.i.d) uncensored data. For more details, see D’Agostino and Stephens (1986).

\subsection{Extended Neyman's Smooth Test (ENST)}

Thomas and Pierce (1979) suggested using power functions instead of using the Legendre polynomials in (2.2) in order to extend Neyman's smooth test to the case of composite hypotheses. In this case, the smooth alternative density (2.2) became of the form

$$
g(u ; \theta)=\exp \left\{\sum_{s=1}^{m} \theta_{s} u^{s}-D(\theta)\right\}
$$

where $m, D(\theta)$, and $u$ are as defined in equation (2.2).

The smooth alternative density (2.4) could be written in terms of the original variable $T$ and its pdf $f(t ; \beta)$, as follows 


$$
g(t ; \beta, \theta)=f(t ; \beta) \exp \left\{\sum_{s=1}^{m} \theta_{s} F^{s}(t ; \beta)-D(\theta)\right\},
$$

where $m$ and $D(\theta)$ are as defined in equation (2.2), and $F(t ; \beta)$ is the CDF corresponding to $f(t$; $\beta)$.

Thomas and Pierce (1979) used partial scores to test for $\theta=0$ which is equivalent to test for (2.1). The testing procedure could be presented as:

Let $T_{1}, T_{2}, \ldots, T_{n}$ be independent continuous random variables. The CDF of $T_{i}$ is given by $F\left(t_{i} ; \beta\right)$, where $\underline{\beta}$ is a vector of $p$ unknown parameters, with corresponding pdf denoted by $f\left(t_{i} ; \beta\right)$. The smooth alternative proposed by Thomas and Pierce (1979) is given by (2.5). They entioned that for some distributions as the exponential and Weibull ones, it is convenient to replace $F(t ; \beta)$ by $\bar{F}(t ; \beta)=1-F(t ; \beta)$, in $(2.5)$ without changing the ultimate test. They used partial scores to test the null hypothesis $H_{0}: \theta=0$ which is equivalent to test the null density $f_{0}(t ; \beta)$ in (2.1). This can be performed by writing the log-likelihood function of the data under (2.5) in the form

$$
\ell(\theta, \beta)=\sum_{i=1}^{n}\left\{\ln f\left(t_{i}, \beta\right)+\sum_{s=1}^{m} \theta_{s} F^{s}\left(t_{i} ; \beta\right)-D(\theta)\right\},
$$

and then getting the partial scores from (2.6) under $H_{0}: \theta=0$ as

$$
\frac{\partial \ell(\theta, \beta)}{\partial \theta_{s}}=\sum_{i=1}^{n}\left\{F^{s}\left(t_{i} ; \beta\right)-\frac{\partial D\left(\theta_{0}\right)}{\partial \theta_{s}}\right\}=\sum_{i=1}^{n}\left\{F^{s}\left(t_{i} ; \beta\right)-(s+1)^{-1}\right\}, s=1,2, \ldots, m,
$$

and

$$
\frac{\partial \ell(\theta, \beta)}{\partial \beta_{w}}=\sum_{i=1}^{n}\left\{\frac{\partial \ln f\left(t_{i}, \beta\right)}{\partial \beta_{w}}\right\}, w=1,2, \ldots, p,
$$

where $\theta 0$ denotes the evaluation at $\theta=0$, and $\frac{\partial D\left(\theta_{0}\right)}{\partial \theta_{s}}=E\left[F^{s}\left(t_{i} ; \beta\right)\right]$

Under $\mathrm{H} 0: \theta=0$, the Fisher information matrix denoted by

$$
I(\theta, \beta)=\left.\operatorname{Cov}\left(\frac{\partial \ell(\theta, \beta)}{\partial \theta}, \frac{\partial \ell(\theta, \beta)}{\partial \beta}\right)\right|_{\hat{\theta}, \hat{\beta}}
$$

partitioned in the form

$$
I(\theta, \beta) \mid=\left(\begin{array}{cc}
I_{\theta \theta}(\theta, \beta) & I_{\theta \beta}(\theta, \beta) \\
I_{\beta \theta}(\theta, \beta) & I_{\beta \beta}(\theta, \beta)
\end{array}\right),
$$

where 


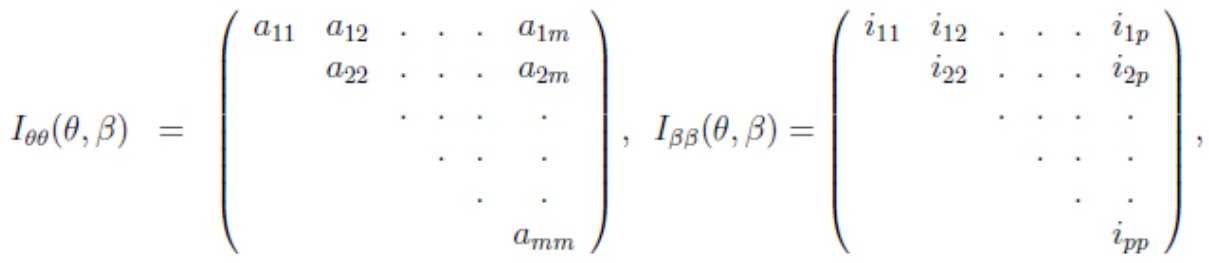

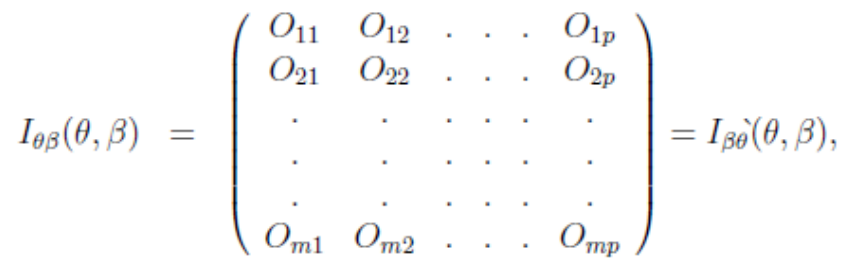

$$
\begin{aligned}
& a_{s h}=-E\left\{\frac{\partial^{2} \ell(\theta, \beta)}{\partial \theta_{s} \partial \theta_{h}}\right\}=\frac{n s h}{(s+h+1)(s+1)(h+1)}, s, h=1,2, \ldots, m, \\
& \frac{\partial^{2} D\left(\theta_{0}\right)}{\partial \theta_{s} \partial \theta_{h}}=\operatorname{Cov}\left(F^{s}\left(t_{i} ; \beta\right), F^{h}\left(t_{i} ; \beta\right)\right) \text {, } \\
& i_{w r}=-E\left\{\frac{\partial^{2} \ell(\theta, \beta)}{\partial \beta_{w} \partial \beta_{r}}\right\}=-n E\left\{\frac{\partial^{2} \ln f\left(t_{i} ; \beta\right)}{\partial \beta_{w} \partial \beta_{r}}\right\}, w, r=1,2, \ldots, p \text {, and } \\
& O_{s w}=-E\left\{\frac{\partial^{2} \ell(\theta, \beta)}{\partial \theta_{s} \partial \beta_{w}}\right\}=-n E\left\{\frac{\partial F^{s}\left(t_{i} ; \beta\right)}{\partial \beta_{w}}\right\}, s=1,2, \ldots, m, w=1,2, \ldots, p .
\end{aligned}
$$

Under $H_{0}: \theta=0$, the quadratic partial score test statistic considered by Thomas and Pierce (1979) takes the form

$$
Q_{m}=\frac{1}{n}\left(\frac{\partial \ell\left(\theta, \hat{\beta}_{0}\right)}{\partial \theta}\right)^{`}\left(n^{-1} I_{\theta \theta \mid \beta}\right)^{-1}\left(\frac{\partial \ell\left(\theta, \hat{\beta}_{0}\right)}{\partial \theta}\right)=\left(\frac{\partial \ell\left(\theta, \hat{\beta}_{0}\right)}{\partial \theta}\right)^{`}\left(I_{\theta \theta \mid \beta}\right)^{-1}\left(\frac{\partial \ell\left(\theta, \hat{\beta}_{0}\right)}{\partial \theta}\right),
$$

where $\frac{\partial \ell\left(\theta, \hat{\beta}_{0}\right)}{\partial \theta}$ is an $(m \times 1)$ vector of the partial scores, $\hat{\beta}_{0}$ is the MLE for $\beta$ when $\theta=0$, and $\mathrm{I}_{\theta \theta \mid \beta}$ is an $(m \times m)$ matrix which represents $\operatorname{Cov}\left(\frac{\partial \ell\left(\theta, \bar{B}_{0}\right)}{\partial \theta}\right)$ and is defined as

$$
I_{\theta \theta \mid \beta}=I_{\theta \theta}-I_{\theta \beta} I_{\beta \beta}^{-1} I_{\beta \theta} .
$$

The asymptotic null distribution of $Q_{m}$ is $\chi_{m}^{2}$ distribution. Thomas and Pierce(1979) derived $n\left(I_{\theta \theta \mid \beta}\right)^{-1}$ for $\mathrm{m}=4$ in the case of exponential, Weibull and normal distributions. They concluded that when the distribution under test is of a location-scale type, $n\left(I_{\theta \theta \mid \beta}\right)^{-1}$ is $\beta$ free, that is; it does not depend on the true values of $\underline{\beta}=(\beta 1, \beta 2, \ldots, \beta p)$. For other distributions, if $n\left(I_{\theta \theta \mid \beta}\right)^{-1}$ depends on $\beta$, one should replace $\beta$ with $\hat{\beta}_{0}$ or use the observed information matrix, without changing the limiting distribution of $Q_{m}$. Thomas and Pierce (1979) used the extended Neyman's smooth test to examine the validity of the exponential, Weibull, and lognormal distributions.

\section{Adapted Extended Neyman's Smooth Test (AENST) and its use in AFT models}


In this Section, the ENST explained in sub-section 2.2 is adapted such that it be-comes valid to be used as a GOF test in AFT models. We call this test; Adapted Extended Neyman's Smooth Test (AENST). The partial scores and the elements of Fisher information matrix are derived for Weibull AFT model in sub-section 3.1, while exponential AFT model is considered as a special case in sub-section 3.2.

\subsection{The case of Weibull AFT model}

Under Weibull inverse power law AFT model, the experiment is conducted according to the following conditions

1. A random sample of size $n$ units are put on test, and all run to failure.

2. There are $k$ test stress levels and $n j$ units are tested at stress level $V j, j=1,2, \ldots, k$.

3. The total number of test units is $n=n 1+n 2+\ldots+n k$.

4. The shape parameter $\eta$ of Weibull distribution is independent of the stress which means that this parameter is the same at each stress level.

5. The stress $V j$ affects the scale parameter, $\alpha j$, through the inverse power law relationship

$$
\varpi_{j}=\frac{C}{V_{j}^{P}}, C>0 .
$$

6. The failure times at stress level $V j, T i j, i=1,2, \ldots, n j, j=1,2, \ldots, k$, are assumed to have Weibull distribution with pdf given by

$$
f\left(t_{i j} ; \varpi_{j}, \eta\right)=\frac{\eta}{\varpi_{j}}\left(\frac{t_{i j}}{\varpi_{j}}\right)^{\eta-1} \exp \left[-\left(\frac{t_{i j}}{\varpi_{j}}\right)^{\eta}\right], t_{i j}>0, \varpi_{j}, \eta>0 .
$$

By substituting (3.1) in (3.2), the pdf of the failure times, $T_{i j}, i=1,2, \ldots, n_{j}, j=1$, $2, \ldots, k$, takes the following form

$$
f\left(t_{i j} ; C, P, \eta\right)=\frac{\eta v_{j}^{P}}{C}\left(\frac{v_{j}^{P} t_{i j}}{C}\right)^{\eta-1} \exp \left[-\left(\frac{v_{j}^{P} t_{i j}}{C}\right)^{\eta}\right], \quad t_{i j}>0, C, \eta>0 .
$$

For convenience, we use the notations $\beta 1=\mathrm{C}, \beta 2=\mathrm{P}$, and $\beta 3=\eta$. In this case, the pdf in (3.3) and the corresponding reliability function have the following forms, respectively

$$
\begin{aligned}
& f\left(t_{i j} ; \beta\right)=\left(\frac{\beta_{3} v_{j}^{\beta_{2}}}{\beta_{1}}\right)\left(\frac{v_{j}^{\beta_{2}} t_{i j}}{\beta_{1}}\right)^{\beta_{3}-1} \exp \left\{-\left(\frac{v_{j}^{\beta_{2}} t_{i j}}{\beta_{1}}\right)^{\beta_{3}}\right\}, t_{i j}>0, \beta_{1}, \beta_{3}>0, \\
& \bar{F}\left(t_{i j} ; \beta\right)=\exp \left[-\left(\frac{v_{j}^{\beta_{2}} t_{i j}}{\beta_{1}}\right)^{\beta_{3}}\right], t_{i j}>0, \beta_{1}, \beta_{3}>0 .
\end{aligned}
$$

Testing for (3.4) is equivalent to test for $\theta=0$ in the smooth alternative given by (2.5) and this is accomplished using the score test. Considering (3.4) and (3.5), the loglikelihood function of the data under (2.5) becomes 


$$
\begin{aligned}
\ell(\theta, \beta) & =\sum_{j=1}^{k} \sum_{i=1}^{n_{j}}\left\{\ln f\left(t_{i j}, \beta\right)+\sum_{s=1}^{m} \theta_{s} \bar{F}^{s}\left(t_{i j} ; \beta\right)-D(\theta)\right\} \\
& =\sum_{j=1}^{k} \sum_{i=1}^{n_{j}}\left\{\ln \left(\frac{\beta_{3} v_{j}^{\beta_{2}}}{\beta_{1}}\right)+\left(\beta_{3}-1\right) \ln \left(\frac{v_{j}^{\beta_{2}} t_{i j}}{\beta_{1}}\right)-\left(\frac{v_{j}^{\beta_{2}} t_{i j}}{\beta_{1}}\right)^{\beta_{3}}\right. \\
& \left.+\sum_{s=1}^{m} \theta_{s} \exp \left[-s\left(\frac{v_{j}^{\beta_{2}}}{\beta_{1}} t_{i j}\right)^{\beta_{3}}\right]-D(\theta)\right\} .
\end{aligned}
$$

The partial scores from (3.6) under $H_{0}: \theta=0$ are given by

$$
\begin{aligned}
\frac{\partial \ell(\theta, \beta)}{\partial \theta_{s}} & =\sum_{j=1}^{k} \sum_{i=1}^{n_{j}}\left\{\bar{F}^{s}\left(t_{i j} ; \beta\right)-\frac{\partial D\left(\theta_{0}\right)}{\partial \theta_{s}}\right\} \\
& =\sum_{j=1}^{k} \sum_{i=1}^{n_{j}}\left\{\exp \left[-s\left(\frac{v_{j}^{\beta_{2}}}{\beta_{1}} t_{i j}\right)^{\beta_{3}}\right]-(s+1)^{-1}\right\}, s=1,2, \ldots, m, \text { and } \\
\frac{\partial \ell(\theta, \beta)}{\partial \beta_{w}} & =\sum_{j=1}^{k} \sum_{i=1}^{n_{j}}\left\{\frac{\partial \ln f\left(t_{i j} ; \beta\right)}{\partial \beta_{w}}\right\} \\
& =\sum_{j=1}^{k} \sum_{i=1}^{n_{j}}\left\{\frac{\partial\left\{\ln \left(\frac{\beta_{3} \beta_{j}^{\beta_{2}}}{\beta_{1}}\right)+\left(\beta_{3}-1\right) \ln \left(\frac{v_{j}^{\beta_{2}} t_{i j}}{\beta_{1}}\right)-\left(\frac{v_{j}^{\beta_{2}} t_{i j}}{\beta_{1}}\right)^{\beta_{3}}\right\}}{\partial \beta_{w}}\right\},
\end{aligned}
$$

for $\mathrm{s}=1,2, \ldots, \mathrm{m}$, and $\mathrm{w}=1,2,3$. The elements of the information matrix $I(\theta, \beta)$ under $H_{0}$ are given by

$$
\begin{aligned}
& a_{s h}=n s h /(s+h+1)(s+1)(h+1), s, h=1,2, \ldots, m, \\
& i_{11}=n\left(\beta_{3} / \beta_{1}\right)^{2}, i_{22}=\beta_{3}^{2} \sum_{j=1}^{k} n_{j}\left(\ln v_{j}\right)^{2}, i_{33}=\left[n / \beta_{3}^{2}\right]\left[1+(1 / 6) \pi^{2}-2 \gamma+\gamma^{2}\right], \\
& i_{12}=\left(-\beta_{3}^{2} / \beta_{1}\right) \sum_{j=1}^{k} n_{j} \ln v_{j}, i_{13}=\left(-n / \beta_{1}\right)(1-\gamma), i_{23}=(1-\gamma) \sum_{j=1}^{k} n_{j} \ln v_{j}, \\
& O_{s 1}=\left[-n s \beta_{3}\right]\left[\beta_{1}(s+1)^{2}\right], O_{s 2}=\left[s \beta_{3} /(s+1)^{2}\right] \sum_{j=1}^{k} n_{j} \ln v_{j}, \text { and } \\
& O_{s 3}=\left[n s / \beta_{3}(s+1)^{2}\right][1-\gamma-\ln (s+1)],
\end{aligned}
$$

where $\gamma=0.5772 \ldots$ is the Euler's constant.

By getting the $I_{\theta \theta \mid \beta}$, it was found that it is $\beta$ free which means that it does not depend on the true values of $\underline{\beta^{`}}=\left(\beta_{1}, \beta_{2}, \beta_{3}\right)$. Thus, under $H_{0}: \theta=0$, the quadratic partial score test statistic is given by 


$$
Q_{m}=\left(\frac{\partial \ell\left(\theta, \hat{\beta}_{0}\right)}{\partial \theta}\right)^{`}\left(I_{\theta \theta \mid \beta}\right)^{-1}\left(\frac{\partial \ell\left(\theta, \hat{\beta}_{0}\right)}{\partial \theta}\right),
$$

where the $s_{t h}$ element of $\frac{\partial \ell\left(\theta, \hat{\beta}_{0}\right)}{\partial \theta}$ can be computed as

$$
\frac{\partial \ell\left(\theta, \hat{\beta}_{0}\right)}{\partial \theta_{s}}=\sum_{j=1}^{k} \sum_{i=1}^{n_{j}}\left\{\exp \left[-s\left(\frac{v_{j}^{\hat{\beta}_{2}} t_{i j}}{\hat{\beta}_{1}}\right)^{\hat{\beta}_{3}}\right]-(s+1)^{-1}\right\} .
$$

In this case, the limiting null distribution of $Q_{m}$ is $\chi^{2}$ distribution with $m$ degrees of freedom. By comparing the p-value of $Q_{m}$ computed from $\chi_{m}^{2}$ distribution with the significance level, the adequacy of Weibull distribution as the parametric model for the lifetime variable at each stress level can be judged.

\subsection{The case of exponential AFT model}

Since, the exponential distribution is a special case of Weibull distribution, then by putting $\eta=1$ in (3.2), the pdf of $T_{i j}, i=1,2, \ldots, n_{j}, j=1,2, \ldots, k$, becomes

$$
f\left(t_{i j} ; \varpi_{j}\right)=\frac{1}{\varpi_{j}} \exp \left[-\left(\frac{t_{i j}}{\varpi_{j}}\right)\right], t_{i j}>0, \varpi_{j}>0 .
$$

Using inverse power law relationship, the pdf in (3.11) takes the following form

$$
f\left(t_{i j} ; \beta\right)=\frac{v_{j}^{\beta_{2}}}{\beta_{1}} \exp \left(-\frac{v_{j}^{\beta_{2}}}{\beta_{1}} t_{i j}\right), t_{i j}>0, \beta_{1}>0 .
$$

Similarly, under $H_{0}: \theta=0$, the quadratic partial score test statistic is given by (3.9).

\section{Conditional Probability Integral Transformation Test (CPITT)}

O'Reilly and Quesenberry (1973) introduced the CPITT that was based on transforming the original set of $n$ random variables into a smaller set of $(n-p)$ - where $p$ is the number of estimated parameters - (i.i.d) Uniform, $U(0,1)$ random variables. Then, test of uniformity can be applied. Abdel-Ghaly et al. (2016) applied this method in the case of AFT model. They assumed that the failure times at each stress level could be transformed separately into i.i.d. $U(0,1)$ random variables. Then the transformed random variables obtained using the CPITT at each stress level are pooled together as one sample hypothesized to be drawn from $U(0,1)$ distribution. Finally, the unifor- mity of the pooled sample and accordingly the adequacy of the hypothesized family of distributions can be judged.

Applying the CPITT using both Weibull and exponential distributions are briefly explained (for more details, see Abdel-Ghaly et al. (2016)) in the following subsections.

\subsection{The case of exponential AFT model}


The failure times, $T_{i j}, i=1,2, \ldots, n_{j}, j=1,2, \ldots, k$ that follow the Weibul distribution in the form (3.4), are transformed into a new set of random variables, $X_{i j}$, where

$$
X_{i j}=T_{i j}^{\beta_{3}}, i=1,2, \ldots, n_{j}, j=1,2, \ldots, k .
$$

In this case, the transformation to $U(0,1)$ is obtained by

$$
\begin{aligned}
U_{i j}=1-\left\{\frac{1-\left(n_{j}-i+1\right) Z_{i j} /\left(Z_{i j}+\ldots+Z_{n_{j} j}\right)}{1-\left(n_{j}-i+1\right) Z_{(i-1) j} /\left(Z_{i j}+\ldots+Z_{n_{j} j}\right)}\right\}^{n_{j}-i}, i & =1,2, \ldots, n_{j}-1, \\
j & =1,2, \ldots, k,
\end{aligned}
$$

where $Z_{i j}$ is the $i^{\text {th }}$ order statistic from the $j^{\text {th }}$ stress level, and $Z_{0 j}=0$.

If the shape parameter $\beta_{3}$ is unknown, it can be replaced by the MLE computed from each stress level separately. This will result in omitting an extra observation when using the CPITT, which in turn leads to replacing $i=1,2, \ldots, n_{j}-1$ by $i=1,2, \ldots, n_{j}-2$ in (4.2). The transformed $(n-2 k) U$ values obtained from all stress levels are pooled together as one sample. The uniformity of the transformed values are tested by using the modified Watson statistic, named $U_{\text {Mod }}^{2}$ (called $U_{\text {Mod-W }}^{2}$ in the case of Weibull distribution). Thus, the assumption of Weibull distribution can be validated.

\subsection{The case of exponential AFT model}

Using $T_{i j M} \dot{\bar{d}} \overline{\bar{d}} 1,2, \ldots, n_{j}, j=1,2, \ldots, k$ with $p d f$ of exponential distribution given by (3.12), the transformed $U$ values from each sub-sample at each stress level are given by (4.2). By testing the uniformity of these $(n-k) U$ values, using the same statistic, $U_{M o d}^{2}$ (called $U_{M o d-E}^{2}$ in the case of exponential distribution), the assumption of exponentiality can be validated.

\section{Simulation Study}

In this Section, the performance of the AENST in validating the underlying distri- butional assumption in AFT models is evaluated. The performance is measured by the size and the power of the test. At each size, the power of both the AENST and CPITT are compared. Testing for both Weibull and exponential distributions are explained in the following subsections.

\subsection{Testing for the Weibull distribution}

In this subsection, the assumption of Weiull distribution in AFT model is to be examined. Firstly, the power of the AENST, $Q_{m}$ given by (3.9) with $m=1$ (called $Q_{1-W}$ in this case) is found. Secondly, the power of the CPITT, $U_{\text {Mod }}^{2}$ (called $U_{\text {Mod }-W}^{2}$ is also given.

The simulation study is conducted under the following experiment

- There are $k=4$ stress levels with different four values: $V_{1}=24, V_{2}=26, V_{3}=28$ and $V_{4}=$ 30 . 
- Four different sample sizes, $n$, and their division on stress levels, $n_{j}, j=1,2,3$, 4, are arbitrary chosen as shown in Table 1 .

- The true values of the parameters of the inverse power law relationship defined in (3.1), $C$ and $P$ are arbitrary chosen to be $(0.5,1.5,3.5)$ and $(0.1,0.5,0.9)$, respectively.Then, nine different combinations of these values are considered.

- The computed values of the variance of the partial score, $I_{\theta \theta \mid \beta}$, needed to calculate the statistic $Q_{1-W}$ are given in Table 2 .

- For each combination of $(C, P, n), 1000$ samples are generated using Mathcad program from

- $\quad$ Exponential distribution with pdf given by (3.11).

- Weibull distribution with pdf given by (3.3), with $\eta=0.5$. The case of $\eta=2$ is also considered, but it is found that there is no much difference between both cases. So, the case of $\eta=0.5$ is the only presented in this paper. When $\eta=1$-which is the case of the exponential distribution- is discussed in subsection 5.2.

- $\quad$ Lognormal distribution with pdf givenby

$$
\begin{array}{r}
f\left(t_{i j} ; C, P, \sigma\right)=\frac{1}{\sigma t_{i j} \sqrt{2 \pi}} \exp \left\{-\frac{\left[\ln t_{i j}-\ln C+P \ln V_{j}\right]^{2}}{2 \sigma^{2}}\right\}, \\
t_{i j}>0, C>0, \sigma>0,
\end{array}
$$

with $\sigma=2$ and the scale parameter, $\exp \left(\mu_{j}\right), j=1,2,3,4$, is expressed by the inverse power law relationship defined by

$$
\exp \left(\mu_{j}\right)=\frac{C}{V_{j}^{P}}, \quad \text { or } \quad \mu_{j}=\ln C-P \ln V_{j}, C>0 .
$$

- For each sample, the MLE of the parameters of these distributions are obtained using Mathcad program with tolerance value $\epsilon=0.00001$. Then, the statistics $\mathrm{Q}_{1-W}$ and $U_{M o d-W}^{2}$ are calculated and the power for each one of them is estimated by

$$
\text { Power }=\text { Number of times rejecting } \mathrm{H}_{0} / 1000 \text {, where }
$$

$\mathrm{H}_{0}$ : $\mathrm{T}_{i j}$ follows Weibull distribution with pdf given by (3.2).

$\mathrm{H}_{1}$ : Not $\mathrm{H}_{0}$.

The estimated power values of $\mathrm{Q}_{1-\mathrm{w}}$ and $U_{M o d-W}^{2}$ statistics are given in Table 3.

From this Table, it is seen that

- In general, The estimated power for $\mathrm{Q}_{1-W}$ statistic increases as the sample sizes increase. For large sample sizes, the power of the this statistic becomes very high and is close to 1 .

- Under the lognormal alternative and for all sample sizes, $\mathrm{Q}_{1-W}$ has observable higher power than $U_{\text {Mod-W }}^{2}$ statistic.

- When testing for the Weibull distribution against Weibull alternative, the estimated power of $\mathrm{Q}_{1-\mathrm{W}}$ is very small, whatever the sample size. This means that there is a very 
small probability to reject $\mathrm{H}_{0}$ when the failure times have already follow Weibull distribution.

- Under the exponential alternative, the estimated power of $\mathrm{Q}_{1-W}$ is not so high. This can be justified by the fact that when the shape parameter of Weibull distribution equals 1 , the distribution is reduced to the exponential one.

- The case when $\mathrm{n}=33$ is not considered when calculating the power of the $U_{M o d-W}^{2}$ statistic because some of the sub-samples at each stress level are very small and using this statistic requires obtaining the MLE of the shape parameter of Weibull distribution at each stress level separately. This problem does not appear when using the $\mathrm{Q}_{1-W}$ statistic.

\subsection{Testing for the exponential distribution}

For the exponential case, to examine the power of the AENST (called $Q_{1-E}$ ), the simulation study is conducted under the same experiment of Weibull case but with different values of the variance of the partial score, $I_{\theta \theta \mid \beta}$, also given in Table 2.

In this case, the hypotheses are given by

$H_{0}: T_{i j}$ follows exponential distribution with pdf given by (3.11).

$H_{1}$ : Not $H_{0}$.

The estimated power values of $Q_{1-E}$ statistic in testing the exponential distribution in AFT model are given in Table 4 under different values of $C, P$, and $n$. From this Table, it is seen that

- The estimated power increases as the sample size increases.

- The $\mathrm{Q}_{I-E}$ statistic is powerful for testing the exponential distribution since the estimated power of this statistic is very high for all sample sizes versus each one of the lognormal and Weibull alternatives. In some cases the power equals 1.

- Comparing with the CPITT test, $U_{M o d-E}^{2}, \mathrm{Q}_{I-E}$ statistic is more powerful for testing H0 versus both the lognormal and Weibull alternatives.

- In the case of Exponential alternative, $\mathrm{Q}_{I-E}$ statistic has a very small power to reject $\mathrm{H} 0$. In the majority of cases, the estimated power of $\mathrm{Q}_{I-E}$ is smaller than that of $U_{M o d-E}^{2}$.

\section{A Real Data Set}

In this Section, we apply the AENST to investigate the distribution of times to breakdown of an insulating fluid. The data is taken from Nair (1982). This data represents the times to breakdown of an insulating fluid under three elevated voltage stresses.

Nelson (1990) suggested using the exponential inverse power law model given by (3.12) to fit the data. To validate this suggestion, the AENST statistic with $m=1, Q_{1-E}$ statistic is computed. The results of applying the test are summarized in Table 5. The MLE of the parameters are obtained using Mathcad program. From Table 5, it is clear that the test gave strong evidence that the exponential inverse power law model is a suitable model to represent the times to breakdown of the insulating fluid given by Nair (1982). 


\section{Conclusions}

In accelerated life testing (ALT), the failure times obtained under high stress con- ditions are used to obtain the lifetime distribution of products under the normal conditions. The incorrect specification of the lifetime distributions of products may result in expensive costs and bad decisions.

The tools used to assess the validity of certain distribution in representing the data are called goodness of fit (GOF) techniques. These techniques are based on measuring the discrepancy of the sample data from the null or the hypothesized distribution.

When dealing with accelerated failure time (AFT) models, it is assumed that a certain model holds. Then, it is desired to test the validity of the underlying distribution. Under constant stress ALT experiment, there are different stress levels. Thus, the failure times at all stress levels should be combined to reach a conclusion about the adequacy of a certain lifetime distribution at each stress level. Thus, there is a need to modify some GOF techniques to be used in this case.

The Neyman's smooth test (NST) is a GOF test that dose not depend on the form of the null density. This test was extended to be used in the case of composite hypotheses. It is called the extended Neyman's smooth test (ENST).

In this paper, the process of adapting the ENST is clarified. The resulting test; which we called the adapted extended Neyman's smooth test (AENST) is then ap- plied to examine the fit of a certain family of distributions under AFT models. We assumed that the AFT model holds and showed how to use the AENST to assess the underlying lifetime distribution. The case of constant stress under complete sam- pling is considered and the method is applied to test for both Weibull and exponential distributions.

A simulation study is conducted to examine the estimated power of the AENST in validating both the Weibull (the test is called $Q_{1-W}$ in this case) and exponential (it is called $Q_{1-E}$ ) distributions when using a certain AFT model. Another GOF test; which is the conditional probability integral transformation test (CPITT) is also applied on the same experiments to the purpose for comparison. The results showed that the estimated powers for both $Q_{1-W}$ and $Q_{1-E}$ statistics increase as the sample sizes increase and for large sample sizes, the power of these statistics become very high and sometimes equal 1 . It is also seen that the power of the AENST is much better than that of the CPITT in the majority of cases. Thus, it is recommended to use the AENST as a GOF test when dealing with AFT models. To summarize, we can say that adapting the ENST will help the development in this field.

Finally, the AENST is applied on a real data set that includes the times to break- down of an insulating fluid to investigate the adequacy of the exponential inverse power law model suggested by Nelson (1990). The results give an evidence that the exponential inverse power law model is suitable to describe the data.

The future work of this study is to modify the AENST in order to be valid to test the underlying distributions in AFT model under step stress ALT. Under step stress, the stress on each unit is increased at pre-specified periods of time, or upon the occurrence of a fixed number of failures. 
Table 1. Total sample sizes $(n)$ and sub-samples $\left(n_{j}\right)$

\begin{tabular}{c|cccc}
\hline & & \multicolumn{3}{|c}{$n_{j}$} \\
\cline { 3 - 4 }$n$ & $n_{1}$ & $n_{2}$ & $n_{3}$ & $n_{4}$ \\
\hline 33 & 3 & 5 & 10 & 15 \\
63 & 13 & 15 & 17 & 18 \\
103 & 18 & 20 & 30 & 35 \\
203 & 35 & 45 & 55 & 68 \\
\hline
\end{tabular}

Table 2. Computed values of $I_{\theta \theta \mid \beta}$ for Weibull and exponential distributions at different $n$

\begin{tabular}{|c|c|c|c|c|}
\hline \multirow[b]{2}{*}{ Distribution } & \multirow[b]{2}{*}{33} & \multicolumn{2}{|c|}{$n$} & \multirow[b]{2}{*}{203} \\
\hline & & 63 & 103 & \\
\hline Weibul & 0.085 & 0.163017 & 0.266520 & 0.525278 \\
\hline Exponentiall & 0.687499 & 1.312500 & 2.145833 & 4.229166 \\
\hline
\end{tabular}


Table 3: Estimated values of the power for testing Weibull distribution

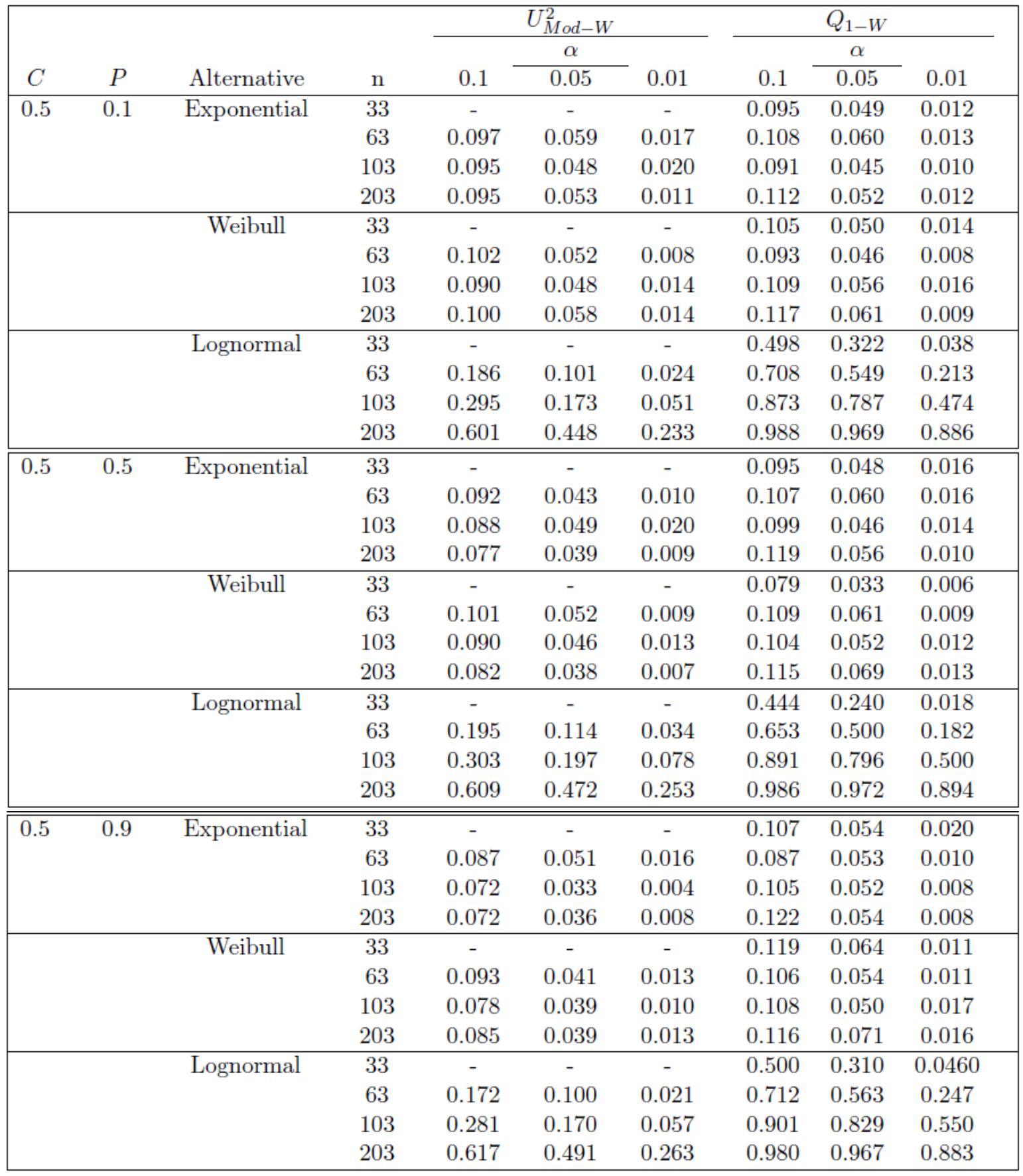


Table 3: Estimated values of the power for testing Weibull distribution (Cont.)

\begin{tabular}{|c|c|c|c|c|c|c|c|c|c|}
\hline \multirow[b]{3}{*}{$C$} & \multirow[b]{3}{*}{$P$} & \multirow[b]{3}{*}{ Alternative } & \multirow[b]{3}{*}{$\mathrm{n}$} & \multicolumn{3}{|c|}{$U_{M o d-W}^{2}$} & \multicolumn{3}{|c|}{$Q_{1-W}$} \\
\hline & & & & & $\alpha$ & & & $\alpha$ & \\
\hline & & & & 0.1 & 0.05 & 0.01 & 0.1 & 0.05 & 0.01 \\
\hline \multirow[t]{12}{*}{1.5} & 0.1 & Exponential & 33 & - & - & - & 0.096 & 0.050 & 0.013 \\
\hline & & & 63 & 0.094 & 0.042 & 0.014 & 0.095 & 0.044 & 0.008 \\
\hline & & & 103 & 0.084 & 0.041 & 0.008 & 0.124 & 0.065 & 0.014 \\
\hline & & & 203 & 0.09 & 0.046 & 0.015 & 0.102 & 0.056 & 0.016 \\
\hline & & Weibull & 33 & - & - & - & 0.094 & 0.045 & 0.011 \\
\hline & & & 63 & 0.095 & 0.046 & 0.003 & 0.091 & 0.050 & 0.010 \\
\hline & & & 103 & 0.083 & 0.038 & 0.006 & 0.108 & 0.054 & 0.010 \\
\hline & & & 203 & 0.069 & 0.035 & 0.010 & 0.120 & 0.062 & 0.010 \\
\hline & & Lognormal & 33 & - & - & - & 0.464 & 0.275 & 0.043 \\
\hline & & & 63 & 0.155 & 0.086 & 0.026 & 0.749 & 0.597 & 0.298 \\
\hline & & & 103 & 0.275 & 0.181 & 0.069 & 0.878 & 0.784 & 0.479 \\
\hline & & & 203 & 0.606 & 0.462 & 0.249 & 0.987 & 0.978 & 0.919 \\
\hline \multirow[t]{12}{*}{1.5} & 0.5 & Exponential & 33 & - & - & - & 0.097 & 0.05 & $\overline{0.014}$ \\
\hline & & & 63 & 0.089 & 0.038 & 0.010 & 0.101 & 0.051 & 0.014 \\
\hline & & & 103 & 0.084 & 0.047 & 0.012 & 0.078 & 0.049 & 0.009 \\
\hline & & & 203 & 0.089 & 0.050 & 0.009 & 0.112 & 0.063 & 0.014 \\
\hline & & Weibull & 33 & - & - & - & 0.076 & 0.031 & 0.007 \\
\hline & & & 63 & 0.089 & 0.038 & 0.010 & 0.112 & 0.065 & 0.014 \\
\hline & & & 103 & 0.074 & 0.040 & 0.011 & 0.101 & 0.059 & 0.013 \\
\hline & & & 203 & 0.079 & 0.033 & 0.009 & 0.124 & 0.068 & 0.013 \\
\hline & & Lognormal & 33 & - & - & - & 0.485 & 0.293 & 0.047 \\
\hline & & & 63 & 0.184 & 0.100 & 0.023 & 0.724 & 0.565 & 0.052 \\
\hline & & & 103 & 0.307 & 0.184 & 0.071 & 0.880 & 0.785 & 0.469 \\
\hline & & & 203 & 0.615 & 0.473 & 0.246 & 0.984 & 0.964 & 0.887 \\
\hline \multirow[t]{12}{*}{1.5} & 0.9 & Exponential & 33 & - & - & - & 0.090 & 0.042 & $\overline{0.016}$ \\
\hline & & & 63 & 0.087 & 0.039 & 0.012 & 0.110 & 0.054 & 0.012 \\
\hline & & & 103 & 0.083 & 0.038 & 0.009 & 0.115 & 0.060 & 0.018 \\
\hline & & & 203 & 0.084 & 0.041 & 0.014 & 0.091 & 0.053 & 0.009 \\
\hline & & Weibull & 33 & - & - & - & 0.107 & 0.048 & 0.014 \\
\hline & & & 63 & 0.090 & 0.052 & 0.010 & 0.122 & 0.072 & 0.013 \\
\hline & & & 103 & 0.106 & 0.049 & 0.013 & 0.113 & 0.064 & 0.013 \\
\hline & & & 203 & 0.073 & 0.035 & 0.007 & 0.113 & 0.072 & 0.012 \\
\hline & & Lognormal & 33 & - & - & - & 0.485 & 0.288 & 0.040 \\
\hline & & & 63 & 0.188 & 0.101 & 0.024 & 0.648 & 0.484 & 0.194 \\
\hline & & & 103 & 0.298 & 0.189 & 0.068 & 0.870 & 0.758 & 0.484 \\
\hline & & & 203 & 0.616 & 0.490 & 0.282 & 0.979 & 0.959 & 0.890 \\
\hline
\end{tabular}


Table 3: Estimated values of the power for testing Weibull distribution (Cont.)

\begin{tabular}{|c|c|c|c|c|c|c|c|c|c|}
\hline \multirow[b]{3}{*}{$C$} & \multirow[b]{3}{*}{$P$} & \multirow[b]{3}{*}{ Alternative } & \multirow[b]{3}{*}{$\mathrm{n}$} & \multicolumn{3}{|c|}{$U_{M o d-W}^{2}$} & \multicolumn{3}{|c|}{$Q_{1-W}$} \\
\hline & & & & & $\alpha$ & & & $\alpha$ & \\
\hline & & & & 0.1 & 0.05 & 0.01 & 0.1 & 0.05 & 0.01 \\
\hline \multirow[t]{12}{*}{3.5} & 0.1 & Exponential & 33 & - & - & - & 0.087 & 0.037 & 0.008 \\
\hline & & & 63 & 0.084 & 0.045 & 0.005 & 0.117 & 0.062 & 0.014 \\
\hline & & & 103 & 0.082 & 0.036 & 0.006 & 0.107 & 0.057 & 0.015 \\
\hline & & & 203 & 0.075 & 0.038 & 0.011 & 0.114 & 0.072 & 0.016 \\
\hline & & Weibull & 33 & - & - & - & 0.099 & 0.048 & 0.012 \\
\hline & & & 63 & 0.085 & 0.041 & 0.010 & 0.087 & 0.044 & 0.006 \\
\hline & & & 103 & 0.084 & 0.041 & 0.006 & 0.116 & 0.062 & 0.010 \\
\hline & & & 203 & 0.085 & 0.032 & 0.006 & 0.126 & 0.062 & 0.016 \\
\hline & & Lognormal & 33 & - & - & - & 0.479 & 0.301 & 0.041 \\
\hline & & & 63 & 0.163 & 0.079 & 0.027 & 0.661 & 0.516 & 0.222 \\
\hline & & & 103 & 0.283 & 0.173 & 0.067 & 0.871 & 0.753 & 0.456 \\
\hline & & & 203 & 0.621 & 0.480 & 0.249 & 0.979 & 0.970 & 0.889 \\
\hline \multirow[t]{12}{*}{ 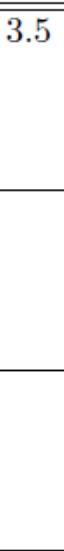 } & 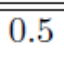 & Exponential & 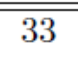 & - & - & - & 0.094 & 0.048 & 0.007 \\
\hline & & & 63 & 0.092 & 0.045 & 0.010 & 0.099 & 0.045 & 0.018 \\
\hline & & & 103 & 0.087 & 0.051 & 0.015 & 0.106 & 0.054 & 0.010 \\
\hline & & & 203 & 0.117 & 0.064 & 0.017 & 0.096 & 0.049 & 0.012 \\
\hline & & Weibull & 33 & - & - & - & 0.094 & 0.048 & 0.010 \\
\hline & & & 63 & 0.099 & 0.048 & 0.012 & 0.111 & 0.070 & 0.013 \\
\hline & & & 103 & 0.084 & 0.044 & 0.017 & 0.100 & 0.059 & 0.016 \\
\hline & & & 203 & 0.080 & 0.038 & 0.008 & 0.123 & 0.062 & 0.015 \\
\hline & & Lognormal & 33 & - & - & - & 0.481 & 0.279 & 0.051 \\
\hline & & & 63 & 0.169 & 0.086 & 0.021 & 0.701 & 0.547 & 0.259 \\
\hline & & & 103 & 0.310 & 0.181 & 0.059 & 0.883 & 0.790 & 0.495 \\
\hline & & & 203 & 0.627 & 0.480 & 0.231 & 0.980 & 0.972 & 0.878 \\
\hline \multirow[t]{12}{*}{3.5} & 0.9 & Exponential & 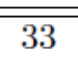 & - & - & - & 0.090 & 0.055 & 0.010 \\
\hline & & & 63 & 0.108 & 0.047 & 0.010 & 0.101 & 0.056 & 0.015 \\
\hline & & & 103 & 0.070 & 0.028 & 0.007 & 0.124 & 0.062 & 0.020 \\
\hline & & & 203 & 0.093 & 0.052 & 0.015 & 0.106 & 0.060 & 0.012 \\
\hline & & Weibull & 33 & - & - & - & 0.109 & 0.059 & 0.014 \\
\hline & & & 63 & 0.084 & 0.053 & 0.019 & 0.117 & 0.059 & 0.007 \\
\hline & & & 103 & 0.087 & 0.040 & 0.005 & 0.120 & 0.059 & 0.012 \\
\hline & & & 203 & 0.088 & 0.033 & 0.007 & 0.126 & 0.066 & 0.016 \\
\hline & & Lognormal & 33 & - & - & - & 0.464 & 0.275 & 0.043 \\
\hline & & & 63 & 0.187 & 0.093 & 0.029 & 0.610 & 0.443 & 0.140 \\
\hline & & & 103 & 0.289 & 0.175 & 0.052 & 0.855 & 0.742 & 0.443 \\
\hline & & & 203 & 0.641 & 0.469 & 0.246 & 0.981 & 0.969 & 0.889 \\
\hline
\end{tabular}


Table 4: Estimated values of the power for testing the exponential distribution

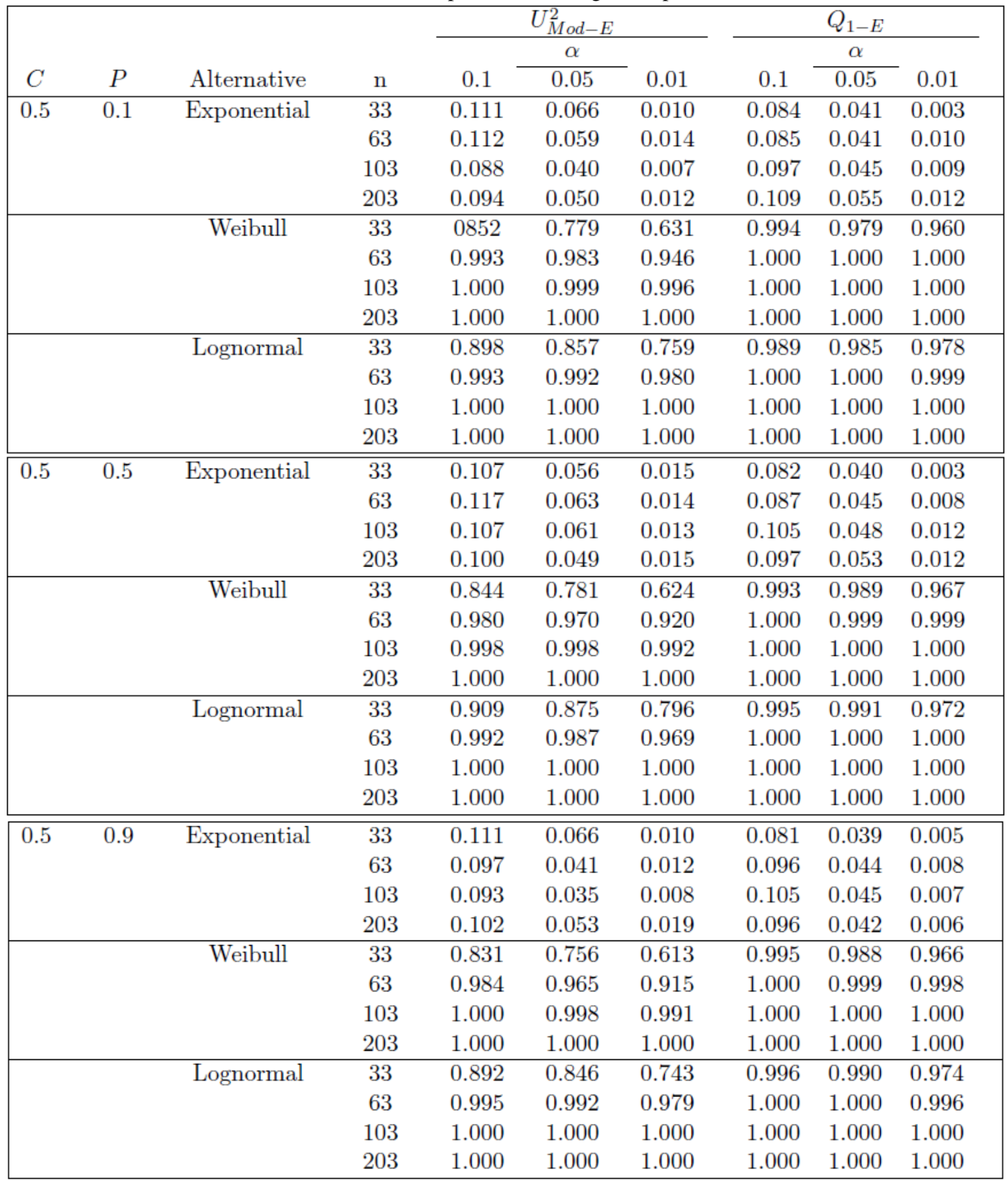


Abdalla Abdel-Ghaly*, Hanan Aly and Elham Abdel-Rahman

Table 4: Estimated values of the power for testing the exponential distribution (Cont.)

\begin{tabular}{|c|c|c|c|c|c|c|c|c|c|}
\hline \multirow[b]{3}{*}{$C$} & \multirow[b]{3}{*}{$P$} & \multirow[b]{3}{*}{ Alternative } & \multirow[b]{3}{*}{$\mathrm{n}$} & \multicolumn{3}{|c|}{$U_{M o d-E}^{2}$} & \multicolumn{3}{|c|}{$Q_{1-E}$} \\
\hline & & & & & $\alpha$ & & & $\alpha$ & \\
\hline & & & & 0.1 & 0.05 & 0.01 & 0.1 & 0.05 & 0.01 \\
\hline \multirow[t]{12}{*}{1.5} & 0.1 & Exponential & 33 & 0.091 & 0.048 & 0.010 & 0.107 & 0.054 & 0.010 \\
\hline & & & 63 & 0.075 & 0.040 & 0.011 & 0.094 & 0.038 & 0.006 \\
\hline & & & 103 & 0.096 & 0.051 & 0.012 & 0.088 & 0.046 & 0.009 \\
\hline & & & 203 & 0.102 & 0.051 & 0.015 & 0.108 & 0.057 & 0.012 \\
\hline & & Weibull & 33 & 0.841 & 0.772 & 0.636 & 0.996 & 0.986 & 0.959 \\
\hline & & & 63 & 0.981 & 0.969 & 0.920 & 1.000 & 1.000 & 0.999 \\
\hline & & & 103 & 1.000 & 1.000 & 0.995 & 1.000 & 1.000 & 1.000 \\
\hline & & & 203 & 1.000 & 1.000 & 1.000 & 1.000 & 1.000 & 1.000 \\
\hline & & Lognormal & 33 & 0.912 & 0.865 & 0.758 & 0.991 & 0.990 & 0.982 \\
\hline & & & 63 & 0.999 & 0.995 & 0.980 & 1.000 & 1.000 & 1.000 \\
\hline & & & 103 & 1.000 & 1.000 & 1.000 & 1.000 & 1.000 & 1.000 \\
\hline & & & 203 & 1.000 & 1.000 & 1.000 & 1.000 & 1.000 & 1.000 \\
\hline \multirow[t]{12}{*}{1.5} & 0.5 & Exponential & 33 & 0.110 & 0.048 & 0.015 & 0.107 & 0.057 & 0.009 \\
\hline & & & 63 & 0.098 & 0.052 & 0.012 & 0.089 & 0.039 & 0.006 \\
\hline & & & 103 & 0.104 & 0.056 & 0.015 & 0.086 & 0.039 & 0.004 \\
\hline & & & 203 & 0.103 & 0.046 & 0.015 & 0.109 & 0.057 & 0.012 \\
\hline & & Weibull & 33 & 0.863 & 0.781 & 0.662 & 0.992 & 0.990 & 0.975 \\
\hline & & & 63 & 0.988 & 0.980 & 0.933 & 1.000 & 1.000 & 1.000 \\
\hline & & & 103 & 1.000 & 1.000 & 0.992 & 1.000 & 1.000 & 1.000 \\
\hline & & & 203 & 1.000 & 1.000 & 1.000 & 1.000 & 1.000 & 1.000 \\
\hline & & Lognormal & 33 & 0.912 & 0.860 & 0.765 & 0.993 & 0.985 & 0.966 \\
\hline & & & 63 & 0.996 & 0.993 & 0.982 & 1.000 & 1.000 & 1.000 \\
\hline & & & 103 & 1.000 & 1.000 & 1.000 & 1.000 & 1.000 & 1.000 \\
\hline & & & 203 & 1.000 & 1.000 & 1.000 & 1.000 & 1.000 & 1.000 \\
\hline \multirow[t]{12}{*}{1.5} & 0.9 & Exponential & 33 & 0.113 & 0.058 & 0.017 & 0.083 & 0.034 & 0.005 \\
\hline & & & 63 & 0.100 & 0.052 & 0.009 & 0.101 & 0.051 & 0.007 \\
\hline & & & 103 & 0.109 & 0.052 & 0.012 & 0.093 & 0.035 & 0.003 \\
\hline & & & 203 & 0.108 & 0.055 & 0.017 & 0.098 & 0.052 & 0.011 \\
\hline & & Weibull & 33 & 0.837 & 0.777 & 0.632 & 0.992 & 0.982 & 0.967 \\
\hline & & & 63 & 0.984 & 0.966 & 0.917 & 1.000 & 0.999 & 0.999 \\
\hline & & & 103 & 1.000 & 0.999 & 0.998 & 1.000 & 1.000 & 1.000 \\
\hline & & & 203 & 1.000 & 1.000 & 1.000 & 1.000 & 1.000 & 1.000 \\
\hline & & Lognormal & 33 & 0.917 & 0.863 & 0.761 & 0.990 & 0.984 & 0.977 \\
\hline & & & 63 & 0.996 & 0.991 & 0.971 & 1.000 & 1.000 & 1.000 \\
\hline & & & 103 & 1.000 & 1.000 & 1.000 & 1.000 & 1.000 & 1.000 \\
\hline & & & 203 & 1.000 & 1.000 & 1.000 & 1.000 & 1.000 & 1.000 \\
\hline
\end{tabular}


Table 4: Estimated values of the power for testing the exponential distribution (Cont.)

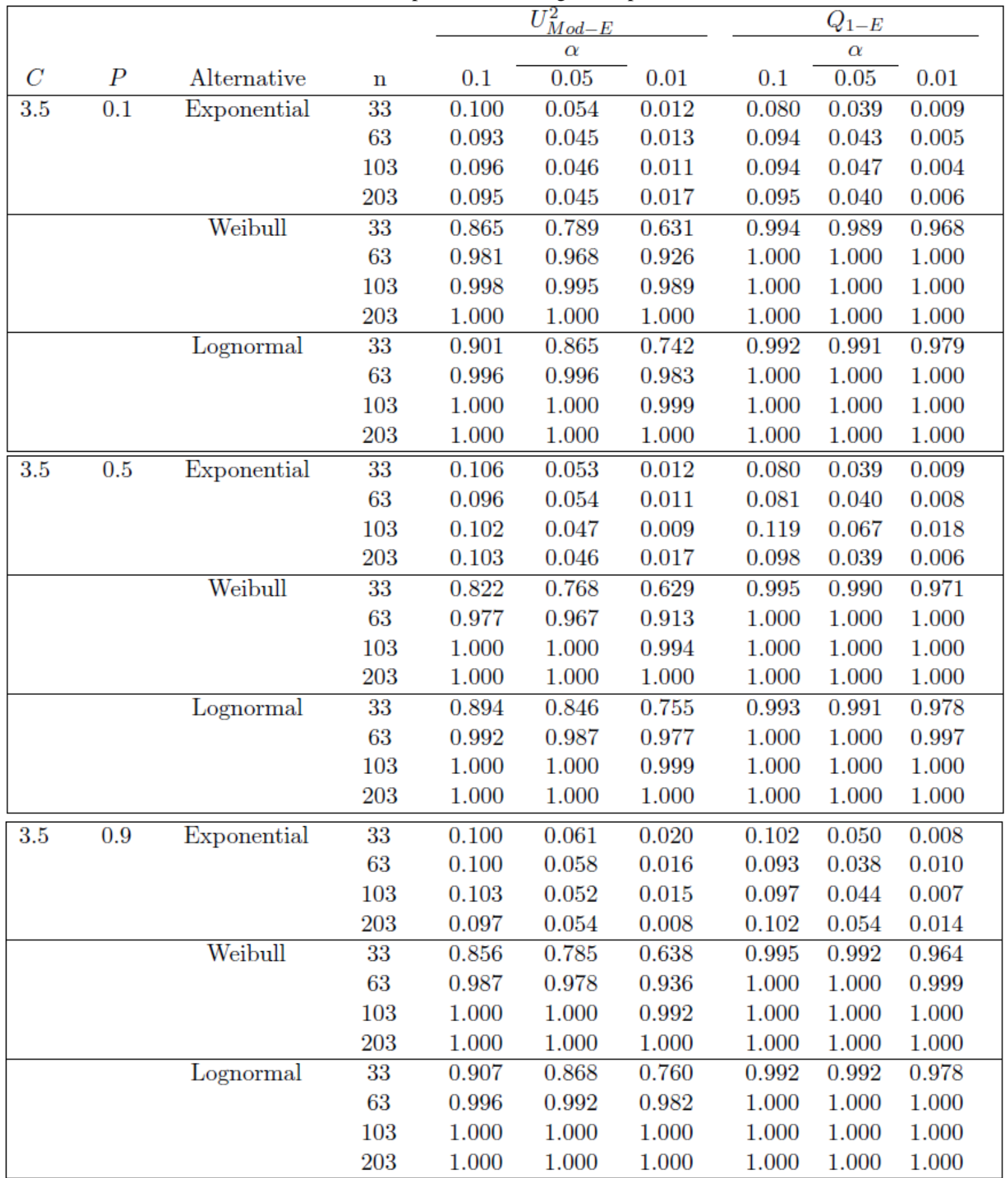


Table 5. The results of applying $\mathrm{Q}_{I-E}$ statistic on the real data set

\begin{tabular}{|c|c|c|c|c|c|}
\hline$\hat{C}$ & $\hat{P}$ & $I_{\theta \theta \mid \beta}$ & $Q_{1-E}$ & $p$-value & $\alpha$ \\
\hline 26.345 & 0.575 & 3.7499955 & 1.443 & 0.23 & 0.05 \\
\hline
\end{tabular}

\section{References}

[1] Abdel Ghaly, A. A., Aly, H. M. and Abdel-Rahman, E. A. (2016). The Use of Conditional Probability Integral Transformation Method for Testing Accelerated Failure Time Models. Pakistan Journal of Statistics and Operation Research. (to appear)

[2] Abdel Ghaly, A. A., Aly, H. M. and Salah, R. N. (2015). Different estimation methods for constant stress accelerated life test under the family of the exponentiated distributions. Quality and Reliability Engineering International, DOI: 10.1002/qre.1818.

[3] Abdel Ghaly, A. A., Attia, A. F. and Aly, H. M. (1998). Estimation of the parameters of Pareto distribution and the reliability function using accelerated life testing with censoring. Communications in Statistics, Simulation and Computation 27, 469-484.

[4] Bagadonavičius, V., Cheminade, O. and Nikulin, M. (2004). Statistical planning and inference in accelerated life testing using the CHSS model. Journal of Statistical Planning and Inference 126, 535-551.

[5] Bagadonavičius, V., Kruopis, J. and Nikulin, M. (2011). Nonparametric Tests for Censored Data. ISTE and J. Wiley, London.

[6] Bagadonavičius, V., Levuliene, R. T. and Nikulin, M. (2013). Chi-squared goodness-of-fit tests for parametric accelerated failure time models, Communications in Statistics - Theory and Methods 42, 2768-2785.

[7] Bagdonavi čius, V. and Nikulin, M. (2002). Accelerated Life Models: Modeling and Statistical Analysis. Champman and Hall/CRC, Boca Raton.

[8] Balakrishnan, N., Chimitova, E., Galanova, N. and Vedernikova, M. (2013). Testing goodness of fit of parametric AFT and PH models with residuals. Communications in Statistics - Simulation and Computation 42, 1352-1367.

[9] D'Agostino and R. Stephens, M. (1986). Goodness-of-Fit Techniques, Marcel Dekker Inc., New York.

[10] Drop, J. R. V. and Mazzuchi, T. A. (2004). A general Bayes exponential inference model for accelerated life testing. Journal of Statistical Planning and Inference 119, 55-74. 
[11] Eguchi, S. (1992). The projection method for accelerated life test model in bivariate exponential distributions. Hiroshima Mathematical Journal 22, 185-193.

[12] Escobar, L. A. and Meeker, W. Q. (2006). A review of accelerated test models, Statistical Science 21, 552-577.

[13] Galanova, N. S., Lemeshko, B. Y. and Chimitova, E. V. (2012). Using nonparametric goodness-of-fit tests to validate accelerated failure time. Optoelectronics, Instrumentation and Data Processing 48, 580-592.

[14] Lawless, J. F. (2003). Statistical Models and Methods for Lifetime Data. John Willey and Sons, New York.

[15] Liu, X. and Tang, L. A (2009). Sequential constant-stress accelerated life testing scheme and its Bayesian inference. Quality and Reliability Engineering International 25, 91-109.

[16] Meeker, W. Q. and Escobar, L. A. (1993). A review of recent research and current issues in accelerated testing. International Statistical Review / Revue Internationale de Statistique 61 $147-168$.

[17] Nair, V. N. (1982). Q-Q plots with confidence bands for comparing several populations. Scandinavian Journal of Statistics 9, 193-200.

[18] Nelson, W. B. (1990). Accelerated Testing: Statistical Models, Test Plans, and Data Analysis. John Wiley and Sons, New York.

[19] Neyman, J. (1937). "Smooth test" for goodness of fit, Skandi-naviske Aktuarietid-dkrift 20, 150-199.

[20] O,Reilly, F. J. and Quesenberry, C. P. (1973). The Conditional Probability Integral Transformation and Applications to Obtain Composite Chi-Square Goodness of Fit Tests. The Annasl of Statistics, 1, 74-83.

[21] Sethuurman, J. and Singpurwalla, N. D. (1982). Testing of hypothesis for distributions in accelerated life testing. Journal of American Statistical Association 77, 204-208.

[22] Teng, S. L. and Yeon, K. P. (2002). A least-squares approach to analyzing life- stress relationship in step-stress accelerated life tests. IEEE Transactions on Reliability 51, 177182.

[23] Thomas, D. R. and Pierce, D. A. (1979). Neyman's smooth goodness-of-fit test when the hypothesis is composite. Journal of the American Statistical Association 74, 441-445. 
[24] Xiong, C. and Ji, M. (2004). Analysis of grouped and censored data From step-stress life test. IEEE Transactions on Reliability 53, 22-28.

[25] Wang, B. X. (2009). Testing for the validity of the assumptions in the exponential step-stress accelerated life-testing model, Computational Statistics and Data Analysis 53, 2702-2709.

Received June 15, 2015; accepted October 10, 2015.

\author{
Abdalla Abdel-Ghaly \\ Faculty of Economics and Political Science \\ Department of Statistics \\ Cairo University \\ abdelghaly@yahoo.com \\ Hanan Aly \\ Faculty of Economics and Political Science \\ Department of Statistics \\ Cairo University \\ han_m_hasan@yahoo.com, hananhasan@feps.edu.eg \\ Elham Abdel-Rahman \\ Faculty of Economics and Political Science \\ Department of Statistics \\ Cairo University \\ ea2006_feps@yahoo.com,ea2006_feps@feps.edu.eg
}


